Cahiers d'études africaines

\title{
Richter, Linda \& Morell, Robert (eds.). - Baba. Men and Fatherhood in South Africa
}

\section{Agnès Martial}

\section{CpenEdition}

1 Journals

Édition électronique

URL : http://journals.openedition.org/etudesafricaines/14485

DOI : 10.4000/etudesafricaines. 14485

ISSN : 1777-5353

Éditeur

Éditions de l'EHESS

\section{Édition imprimée}

Date de publication : 28 mai 2013

Pagination : 488-492

ISBN : 978-2-7132-2387-7

ISSN : 0008-0055

\section{Référence électronique}

Agnès Martial, « Richter, Linda \& Morell, Robert (eds.). - Baba. Men and Fatherhood in South Africa », Cahiers d'études africaines [En ligne], 209-210 | 2013, mis en ligne le 27 mai 2013, consulté le 24 septembre 2020. URL : http://journals.openedition.org/etudesafricaines/14485 ; DOI : https://doi.org/ 10.4000/etudesafricaines. 14485

Ce document a été généré automatiquement le 24 septembre 2020.

(c) Cahiers d'Études africaines 


\title{
Richter, Linda \& Morell, Robert (eds.). - Baba. Men and Fatherhood in South Africa
}

\author{
Agnès Martial
}

\section{RÉFÉRENCE}

RICHTER, Linda \& MORELL, Robert (eds.). - Baba. Men and Fatherhood in South Africa. Cape Town, Human Sciences Research Council Press, 2006, 416 p., bibl.

1 «Baba » est en langue zulu un terme de politesse utilisé à l'attention des hommes plus âgés. Suggérant l'existence d'un rapport quasi filial emprunt de respect, de proximité et de protection, cette appellation traditionnelle revalorise des relations paternelles profondément mises à mal en Afrique du Sud. Elle est aussi d'une grande souplesse, recouvrant une pluralité de figures paternelles bien au-delà de la stricte paternité «biologique». une telle dénomination permet de renouer avec l'organisation traditionnelle de la parenté dans les sociétés de langue zulu et de rappeler l'importance de la paternité au regard des enjeux familiaux, sociaux et de santé publique en Afrique du Sud.

2 Issu d'un programme communautaire, le «Fatherhood Project », initié en 2003 par le Child Youth and Family Development Program at the Human Sciences Research Council, cet ouvrage a pour objectif d'analyser, promouvoir et restaurer le rôle des hommes et des pères dans la famille et la société sud-africaines. Vingt-trois contributions développant différentes approches disciplinaires (histoire, anthropologie, sociologie, démographie, psychologie, droit) y analysent l'histoire politique, économique et sociale de la paternité en Afrique du Sud, explorant l'évolution des représentations de la paternité et de la masculinité au regard de la dynamique des rapports de sexes et de races, les changements récents des normes juridiques et des modes de vie conjugale et familiale, et les enjeux nouveaux que 
suscite, autour du rôle des hommes et des pères, l'épidémie de VIH. Divisé en cinq parties (1. «Opening lines»; 2. «Fatherhood in historical perspective»; 3. "Representations and roles»; 4. "Being a father in South Africa today"; 5. "Local and international policies and programmes »), l'ouvrage utilise les apports théoriques et conceptuels des recherches sur la paternité et la masculinité (en sciences sociales et en psychologie) replacées dans le contexte sud-africain. Différentes contributions analysent l'image des hommes en tant que pères dans les médias et les œuvres de fiction (littérature et cinéma), les voies et les obstacles juridiques cernant la redéfinition de la paternité, les situations concrètes dans lesquelles les hommes expérimentent aujourd hui leur rôle de père. La dernière partie présente les actions gouvernementales et les initiatives issues de la société civile en faveur de la paternité, de l'égalité de genre, et de l'engagement des hommes auprès des femmes et des enfants.

3 Au principe de cet ouvrage se dessine un constat: la majorité des enfants noirs sudafricains grandissent aujourd'hui en l'absence de leur père. Dorrit Posel et Richard Devey, tout en pointant l'insuffisance des données statistiques sud-africaines sur cette question, évaluent ainsi à $63 \%$ de l'ensemble des enfants d'origine africaine le nombre de ceux qui vivent dans un foyer dont le père est absent. Différents auteurs éclairent cette situation en puisant à l'histoire politique et sociale de l'Afrique du Sud, décrivant les effets dévastateurs du système colonial puis de l'apartheid sur les structures sociales et familiales traditionnelles. Les contributions de Mark Hunter et Francis Wilson rappellent ainsi que la paternité représentait dans les sociétés de langue zulu l'axe central de l'accomplissement d'un homme et la justification de sa place sociale, comme symbole et gardien du pouvoir dans la famille et la communauté. Elle trouvait sens au sein d'une organisation clanique patriarcale où le rôle social de père était entrelacé avec celui de chef de la maison (umnuzana), leader et protecteur du foyer (umuzi), garant de la perpétuation du lignage. Paternités biologique (fathering) et sociale (fatherhood) étaient articulées dans l'ubudoa, ambition pour chaque homme de construire et d'assumer la responsabilité d'un foyer. Le rôle du père comme pourvoyeur et responsable de la subsistance de la famille étendue impliquait en outre de payer le prix de la fiancée (ilobolo) pour les fils et de marier les filles. Si le père, en tant que détenteur de l'autorité dans la famille et la communauté, offrait aux jeunes garçons un modèle d'identification, la socialisation quotidienne des enfants revenait plutôt au groupe des pairs ou des garçons plus âgés : la paternité, non réductible à sa dimension biologique, est dans les sociétés zulu une catégorie fluide incluant notamment les oncles paternels, frères aînés (elder father, big father) ou cadets (younger father, little father) du père.

Instaurée dès la période coloniale, la division raciale du marché du travail contraint cependant les hommes à la migration (Francis Wilson, Mamphela Ramphele, Linda Richter): en zone rurale, les pères quittent leur famille pour gagner leur vie, les contacts étant fréquemment réduits à des visites annuelles. Se développent ainsi très tôt des formes de paternité où ne domine plus qu'un rôle de pourvoyeur et de responsable distant, les mères élevant seules leurs enfants (Slani Ngobeni). Dans les villes, l'urbanisation et la création des townships transforment les contours matériels de la maisonnée, conçue sur le modèle occidental de la famille nucléaire et qui ne peut accueillir la famille étendue traditionnelle. Les déplacements forcés de population, séparant les membres de la parenté, contribuent aussi à défaire les liens sociaux traditionnels. Comme le montre Linday Clowes à partir de l'analyse du magazine Drum, l'image des hommes africains dans les médias et la publicité tend en outre, à la fin des années 1950, à délaisser une définition profondément relationnelle de la masculinité, 
inscrite dans la sphère privée et familiale, pour se conformer au modèle «blanc », qui isole le père, en tant que pourvoyeur, au sein du monde professionnel.

Partout, le statut de pourvoyeur est cependant menacé par la pauvreté qui frappe les populations noires du fait de la dépossession économique et foncière, puis de l'explosion du chômage (Linda Richter, Mamphela Remphele, Mark Hunter, Francis Wilson). Privés de la capacité à se marier en s'acquittant de l'ilobolo, de nombreux hommes ne peuvent plus assumer leur rôle social de pères et de pourvoyeur. Mark Hunter signale ainsi que moins de $30 \%$ des Africain(e)s de plus de 15 ans sont aujourd'hui marié(e)s en Afrique du Sud.

D autres évolutions, d'ordre politique, contribuent à défaire les pères de leur autorité : l'évolution des relations de genre et la lutte pour la libération des femmes ont mis en cause le rôle masculin de chef de famille. La résistance et la lutte contre l'apartheid, qui auraient pu revaloriser la paternité, ont surtout été le fait des jeunes hommes qui s'emparaient ainsi de l'autorité politique auparavant réservée à leurs pères (Desmond Lesejane).

$7 \quad$ La masculinité et les valeurs qui lui sont attachées sont profondément affectées par ces évolutions (Francis Wilson, Robert Morell). Il ne reste du système patriarcal ancien que l'abus de pouvoir et la domination, sans le respect des obligations qui lui correspondait. Mark Hunter décrit ainsi une relation ambiguë des hommes à la paternité : masculinité et virilité y sont partiellement assurées par le fait de séduire une ou plusieurs partenaires et de concevoir de nombreux enfants, tout en étant fragilisées par l'incapacité des hommes à remplir les devoirs associés au rôle paternel; nombre d'entre eux ne voient pas l'intérêt de maintenir un lien durable avec les mères de leurs enfants et quand ils le souhaitent, l'insuffisance de leurs ressources économiques les en empêche. Masculinité et paternité sont fréquemment associées à l'abus d'autorité et à la violence envers les femmes et les enfants. Les médias développent une image de masculinité belliqueuse, où la paternité est rarement mise en scène, et qui offre à des enfants vivant sans référent paternel un piètre modèle d'identification (Jeanne Prinsloo). Dans un tel contexte, de nombreuses femmes préfèrent les unions de court terme et la monoparentalité à la présence quotidienne d'un homme instable, irresponsable, voire menaçant.

8 Cette fragilisation du rôle des hommes, en tant que conjoints et pères, recèle des enjeux cruciaux dans le contexte d'épidémie de $\mathrm{VIH}$ qui ravage les familles et touche particulièrement les mères de jeunes enfants, groupe le plus vulnérable et le plus affecté par l'épidémie. Le phénomène grandissant des orphelins du sida renvoie à l'absence des pères : parmi les pays africains examinés, l'Afrique du Sud a le taux le plus bas d'enfants orphelins de mère vivant avec le parent survivant. Séparations et décès laissent un nombre croissant d'enfants sans aucun parent pour les prendre en charge ; le fardeau du soin aux enfants et aux proches repose alors sur la parenté et principalement sur les femmes (tantes et grands-mères). Les auteurs soulignent ainsi l'urgence d'une mobilisation et d'un engagement des hommes, pères biologiques ou "de substitution" - beaux-pères, oncles ou frères aînés - auprès des enfants orphelins (Chris Desmond, Cos Desmond, Philipe Denis et Radikobo Ntsimane).

9 L'ouvrage dresse alors les contours d'une nouvelle paternité, puisant aux valeurs culturelles traditionnelles mais aussi aux principes contemporains de l'égalité de genre : il ne s'agit pas de prôner un retour à la norme patriarcale à partir d'une vision romantique et idéalisée de la paternité ancienne (Desmond Lesejane). En outre, comme 
le souligne Solani Ngobeni à partir de l'analyse critique du film américain Boyz'N the Hood, la dénonciation de l'absence des hommes et de ses effets potentiellement négatifs sur le bien-être des enfants ne doit pas conduire à essentialiser la figure paternelle, en occultant l'immense contribution des femmes à l'éducation des enfants, voire en dévalorisant celle-ci. L'image du père qui guide, protège, procure des soins et de l'attention à sa famille doit être restaurée, à condition qu'elle respecte les valeurs d'égalité de genre désormais inscrites dans la constitution sud-africaine.

Les concepts et travaux relatifs aux nouveaux principes et usages de la paternité sont ainsi mobilisés. Dans leur grande majorité, ces études ont porté sur les familles blanches de classes moyennes et supérieures, en Europe comme en Amérique du Nord. Ces travaux informent bien la situation des pères divorcés d'une partie de la population sud-africaine, correspondant aux mêmes caractéristiques sociologiques, comme en témoigne la contribution de Grace Khunou concernant les procédures judiciaires de divorces dans lesquelles des hommes (blancs) contestent des normes de genre qui les réduisent au rôle de simples pourvoyeurs en limitant leurs droits d'accès à l'enfant.

11 On peut cependant se demander, à l'instar de Robert Morell, comment appréhender la diversité des conceptions de la masculinité et de la paternité dans un pays regroupant des populations dont l'histoire, les traditions culturelles et les conditions de vie sont fort différentes, les distinctions sociales recoupant aujourd hui les anciennes divisions raciales. Du père pourvoyeur, autoritaire et protecteur au «nouveau père » donneur de soins, démonstratif et affectueux, il importe de tenir compte d'une pluralité d'interprétations contribuant ensemble au processus global de redéfinition de la paternité. Différentes contributions s'intéressent ainsi à la révision juridique des droits et devoirs des pères, notamment naturels, et aux politiques gouvernementales en faveur de la paternité (Jacqui Gallinetti, Alan Hosking), aux conceptions et attentes enfantines à l'égard des pères (Linda Richter, Wendy Smith), aux relations quasi paternelles nouées au sein des institutions accueillant des jeunes en difficulté (Azeem Badroodien), ou au point de vue des hommes travaillant dans les mines d'or, pères résidents ou non résidents, sur leurs relations aux enfants (Marlize Rabe).

Linda Richter et d'autres auteurs dans cet ouvrage valorisent les indices d'une nouvelle dynamique de redéfinition des rapports de genre. Liée à l'augmentation de la présence des femmes sur le marché du travail, elle se traduit aussi par la volonté de certains jeunes hommes de s'investir plus fortement dans l'éducation de leurs enfants. Différents programmes gouvernementaux, ainsi que des mouvements tels que le South African Mens' Forum ou le Moral Regeneration Movement, parfois inspirés de mouvements européens comme Fathers' Direct au Royaume-Uni, tentent d'impulser une dynamique d'égalité de genre et un engagement croissant des pères dans la vie familiale (Dean Peacock, Mbuyiselo Botha, Patrice Engle, Tom Beardshaw, Craig R. Loftin), développant ainsi de nouvelles conceptions de la paternité et de la masculinité.

Une fois reconnu ce principe d'égalité de genre, Desmond Lesejane et Nhlanhla Mkhize proposent de reconstruire une conception commune du rôle paternel, collectivement négociée et replacée dans la dimension éminemment relationnelle des organisations sociales africaines traditionnelles. Il s'agirait alors de promouvoir les principes d'autorité, de responsabilité, de soin et d'attention aux proches, de reconnaître la pluralité sociale des figures paternelles présentes autour des enfants et de tenir compte de la diversité des relations concrètes, inscrites ou non dans la corésidence, des pères et des enfants. La redéfinition de la paternité pourrait de la sorte contribuer à la 
construction d'une "masculinité pacifique, démocratique, tolérante et respectueuse " (Robert Morell). Ce livre démontre ainsi le caractère central de la paternité dans la vie des hommes et dans les expériences des enfants, et défend l'idée que les hommes peuvent apporter une contribution majeure au mieux-être de la société sud-africaine en prenant soin d'une nouvelle génération d'enfants, pour qui les pères compteront plus par leur présence que par leur absence (Linda Richter, Robert Morell). S'inventent alors, dans un contexte extrêmement difficile, de nouvelles manières d'être père et de concevoir la paternité, et c'est tout l'intérêt de ce foisonnant ouvrage. 